\title{
Tissue plasminogen activator and seizures: a clot-buster's secret life
}

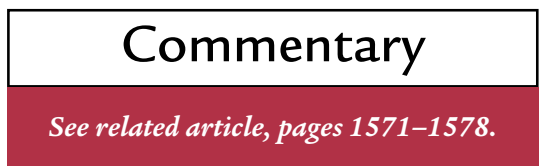

\author{
Robert Pawlak and Sidney Strickland \\ Laboratory of Neurobiology and Genetics, The Rockefeller University, New York, New York, USA \\ Address correspondence to: Sidney Strickland, Laboratory of Neurobiology and Genetics, \\ The Rockefeller University, New York, New York 10021, USA. \\ Phone: (212) 327-8705; Fax: (212) 327-8774; E-mail: strickland@rockefeller.edu.
}

J. Clin. Invest. 109:1529-1531 (2002). doi:10.1172/JCI200215961.

Seizures, caused by hypersynchronized electrical activity, can occur when an area of the brain becomes injured as a result of various pathologies such as degenerative disorders, brain tumors, or head trauma. Seizures are an extremely widespread problem, with about $10 \%$ of the population experiencing them at least once in their lives. They are hazardous both because of the uncontrolled motor activity they generate and because they can cause neuronal death and in some cases permanent neurological deficits (1).

Phenytoin and related antiseizure agents act by prolonging the refractory period of the action potential. This extension prevents the neurons from entering into the rapid firing associated with seizures but allows normal conduction to occur. These drugs generally have a narrow therapeutic range and often have undesirable side effects even when the dosage is optimized.
Therefore, a better understanding of seizure propagation might lead to insights about new therapies, and also perhaps a better understanding of normal neuronal activity.

\section{Tissue plasminogen activator in seizures}

Numerous factors contributing to the generation or facilitation of seizures have been identified, most of which also control physiological neuronal activity. These include excitatory or inhibitory amino acids such as glutamate or GABA, and numerous peptide neurotransmitters (2). In 1993, Qian et al. identified a new player in the seizure arena when they found that tissue plasminogen activator (tPA), a protease best known for its clot-busting ability, is induced within 1 hour after the onset of metrazol-induced seizures (3). Other work showed that the increase in extracellular tPA activity after convulsants could be even more rapid, since the enzyme can be stored in neurons and released upon depolarization (4-6).

The correlation of tPA induction with seizure suggested that the protease might initiate or propagate this event, and indeed, Tsirka et al. $(7,8)$ found that the seizure severity after injection of convulsants is reduced in $t P A^{-/-}$mice. In keeping with the association between seizures and neuronal death, $t P A^{-/-}$mice are also resistant to neurodegeneration after injection of excitotoxin (7).

These results immediately raised the question of how a presumably extracellular protease could affect electrical activity and cell death. The recognized physiological role for tPA is to activate the zymogen plasminogen, which is present in high concentration in blood and many extracellular fluids. tPA can cleave a single arginine-valine bond in

\section{Figure 1}

The distribution of tPA and its role in the hippocampus. (a) tPA immunohistochemistry (red) with superimposed scheme of synaptic input from the amygdala and the three-synaptic hippocampal neuronal circuit (yellow). tPA immunoreactivity is observed almost exclusively in the mossy fiber pathway (24), suggesting that it plays a particularly important role in conducting impulses from the mossy fibers to the Schaffer collaterals. Neuronal cell bodies are counterstained with 4,6diamino-2-phenylindole (blue). CA1 and CA3, regions of the hippocampus; DG, dentate gyrus. (b) Schematic representation of a hippocampal synapse, showing the putative mechanism of tPA action at that site. TPA (gray) is released from the axon terminal upon neuronal depolarization, where it facilitates spreading of an impulse to the postsynaptic site by activating NMDA receptors (green). During excitotoxic challenge, excessive amounts of tPA could also activate plasminogen (blue) to trigger neuronal death by degrading laminin (11). tPA activity could be inhibited by neuroserpin (yellow), thus attenuating seizure spreading and cell death.

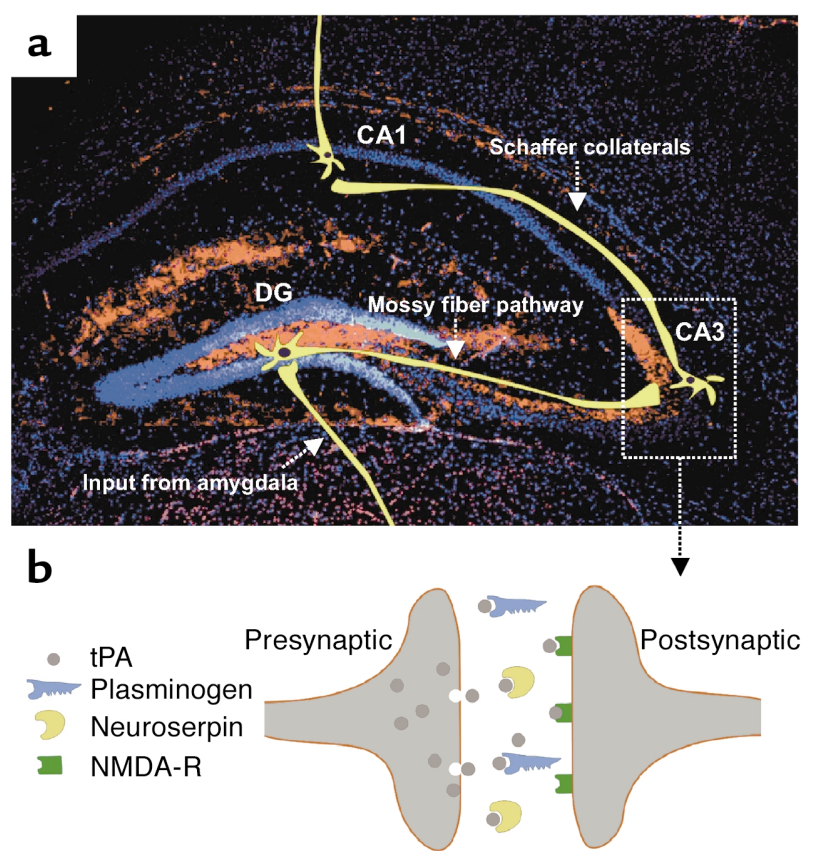




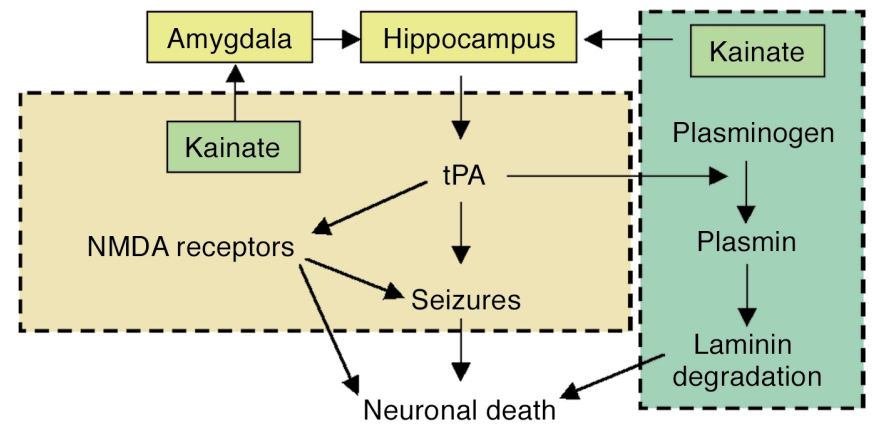

Figure 2

Hypothetical and simplified distinction between tPA-mediated seizures and neuronal death. tPA could facilitate seizures by a plasminogen-independent mechanism involving activation of NMDA receptors. For neuronal death, tPA could act either through a plasminogen-dependent pathway to catalyze degradation of laminin, or via NMDA receptors. Interactions between the two mechanisms are possible but are not shown here.

plasminogen, converting the inactive form into the broad-spectrum serine protease plasmin (9). Thus, tPA and plasminogen can constitute a twomember protease cascade, and a cell that secretes $\mathrm{tPA}$ can generate substantial proteolytic activity via plasmin generation. If tPA neurotoxicity occurs exclusively via activation of plasminogen, it would be expected that Plasminogen $^{-/-}$mice would exhibit a phenotype similar to and at least as pronounced as that of $t P A^{-/-}$mice. In the first neuronal model to be tested in this manner excitotoxin injection into the hippocampus - these two genotypes confer a similar resistance to cell death (10). Other biochemical experiments have confirmed that tPA contributes to neuronal death via activation of plasminogen in this case $(8,10,11)$.

\section{tPA has neuronal effects independent of plasminogen activation}

However, the plot soon thickened. In experiments on a mouse model of stroke, $\mathrm{PP}^{-/-}$mice were resistant to neuronal death $(12,13)$, whereas Plasminogen ${ }^{-/}$mice had exacerbated damage (13). Other threads began to be added to the fabric of plasminogen-independent roles for tPA: maximal activation of microglia depended on tPA but did not require its proteolytic activity (14), mossy fiber outgrowth in the mouse hippocampus was reduced in the absence of tPA but normal in the absence of plasminogen (15), and tPA enhancement of NMDA receptor signaling by cleavage of its NR1 subunit occurred in the absence of plasminogen (16). These results demonstrated that plasminogen-dependent and plasminogen-independent effects of tPA could be experimentally dissociated, strengthening the argument that tPA plays normal and pathological roles that do not require plasminogen activation.

The paper by Yepes et al. (17) in this issue of the JCI adds to this growing body of work. Their experimental protocol was to stimulate seizures using the excitotoxin kainate, a potent glutamate analog that can cause massive depolarization and cell death in neurons. Kainate was delivered into the amygdala, a part of the limbic system that also includes the hippocampal formation (hippocampus and dentate gyrus), a major center for declarative memory. The circuitry of the limbic system has been described in detail and involves propagation of impulses from the amygdala to the dentate gyrus and then to the hippocampus (Figure 1). Thus, hyperstimulation of the amygdala and subsequent examination of the hippocampus allow evaluation of effects caused by electrical activity, and not by direct application of the excitotoxin.

Injection of kainate into the amygdala caused upregulation of $\mathrm{PA}$ in the amygdala and hippocampus, followed by seizures and hippocampal neuronal death (17). Consistent with a plasminogen-independent effect of tPA on electrical activity in this setting, the propagation of seizures was attenuated in $\mathrm{tPA}^{-/-}$mice but not in Plasmino$\mathrm{gen}^{-/-}$mice. Yepes et al. then showed that injection into the hippocampus of neuroserpin, a neuron-specific serine-protease inhibitor that efficiently inhibits tPA, attenuates the seizures (17). Hence, hippocampal tPA appears to modulate the electrical activity generated by kainate injection into the amygdala by a mechanism independent of plasminogen activation.

\section{tPA may facilitate seizures and neuronal death via different mechanisms}

How can this plasminogen-independent effect of tPA on kainate/amygdala-induced seizures be reconciled with previous evidence (7) implicating both tPA and plasminogen in kainate/hippocampus-induced neuronal death? Setting aside the various methodological differences between these studies, it may be that tPA uses distinct mechanisms and acts on different substrates in facilitating seizure and in promoting cell death, as proposed in Figure 2. For cell death, massive depolarization of neurons could lead to high levels of tPA activity, which in turn could produce plasmin. This protease can cause neuronal death via degradation of the ECM molecule laminin (11). In contrast, seizures could be facilitated by tPAmediated cleavage of the NMDA receptor NR1 subunit (16), leading to excessive electrical activity.

For clinical purposes, the current experiments raise the question of whether the rodent model used is relevant to human seizures. In this regard, it is striking that patients with a point mutation in the gene for neuroserpin are subject to seizures (18), perhaps because of tPA hyperactivity in the absence of this proteinase inhibitor. The fact that tPA can facilitate seizures and neuronal death in rodents also raises clear concerns about the use of this clot-buster in ischemic stroke patients, especially since about $10 \%$ experience seizures. Various animal stroke models have yielded conflicting results about the effects of $\mathrm{tPA}$; in some experiments, tPA was beneficial, whereas in others it was deleterious (reviewed in ref. 19). One interpretation that can reconcile many of these conflicts is that tPA has dual roles, each of which is critical at a different time of stroke progression. Thus, in the event of an obstructed blood vessel, dissolution of the clot catalyzed by tPA and restoration of blood flow would be beneficial, but subsequent leakage of tPA into the brain parenchyma could 
have unwanted effects including neuronal death. Therefore, tPA could appear beneficial or deleterious in stroke models, depending on which of these functions predominates. It follows from this interpretation that an ideal stroke therapeutic would be a molecule with robust fibrinolytic ability but with little capacity for inducing neuronal death.

The tPA produced by limbic neurons and secreted upon depolarization (4-6) exerts multiple effects in the nervous system. It has been implicated in normal neuronal activities such as longterm potentiation $(20,21)$ and, most recently, learning and memory $(22,23)$. The consistent finding that exaggerated tPA activity in disease and injury can lead to seizures and neuronal death opens up an opportunity to learn more about these pathologies and develop new approaches to their therapy.

\section{Acknowledgments}

We thank Jerry Melchor and Fernando Sallés for critical reading of this Commentary. The research in our laboratory is supported by NIH grants NS-35704 and NS-38472, and a grant from the Institute for the Study of Aging.

1. Wasterlain, C.G., and Shirasaka, Y. 1994. Seizures, brain damage and brain development. Brain Dev. 16:279-295

2. Glass, M., and Dragunow, M. 1995. Neurochemical and morphological changes associated with human epilepsy. Brain Res. Brain Res. Rev. 21:29-41.
3. Qian, Z., Gilbert, M.E., Colicos, M.A., Kandel, E.R., and Kuhl, D. 1993. Tissue-plasminogen activator is induced as an immediate-early gene during seizure, kindling and long-term potentiation. Nature. 361:453-457.

4. Gualandris, A., Jones, T.E., Strickland, S., and Tsirka, S.E. 1996. Membrane depolarization induces calcium-dependent secretion of tissue plasminogen activator. J. Neurosci. 16:2220-2225.

5. Baranes, D., et al. 1998. Tissue plasminogen activator contributes to the late phase of LTP and to synaptic growth in the hippocampal mossy fiber pathway. Neuron. 21:813-825.

6. Parmer, R.J., et al. 1997. Tissue plasminogen activator $(\mathrm{t}-\mathrm{PA})$ is targeted to the regulated secretory pathway. Catecholamine storage vesicles as a reservoir for the rapid release of t-PA.J. Biol. Chem. 272:1976-1982.

7. Tsirka, S.E., Gualandris, A., Amaral, D.G., and Strickland, S. 1995. Excitotoxin-induced neuronal degeneration and seizure are mediated by tissue plasminogen activator. Nature. 377:340-344.

8. Tsirka, S.E., Rogove, A.D., and Strickland, S 1996. Neuronal cell death and tPA. Nature. 384:123-124.

9. Plow, E.F., Herren, T., Redlitz, A., Miles, L.A., and Hoover-Plow, J.L. 1995. The cell biology of the plasminogen system. FASEB J. 9:939-945.

10. Tsirka, S.E., Rogove, A.D., Bugge, T.H., Degen, J.L., and Strickland, S. 1997. An extracellular proteolytic cascade promotes neuronal degeneration in the mouse hippocampus. J. Neurosci. 17:543-552.

11. Chen, Z.L., and Strickland, S. 1997. Neuronal death in the hippocampus is promoted by plasmin-catalyzed degradation of laminin. Cell. 91:917-925

12. Wang, Y.F., et al. 1998. Tissue plasminogen activator ( $\mathrm{tPA}$ ) increases neuronal damage after focal cerebral ischemia in wild-type and tPA-deficient mice. Nat. Med. 4:228-231.

13. Nagai, N., De Mol, M., Lijnen, H.R., Carmeliet, P., and Collen, D. 1999. Role of plasminogen system components in focal cerebral ischemic infarction: a gene targeting and gene transfer study in mice. Circulation. 99:2440-2444.

14. Rogove, A.D., Siao, C., Keyt, B., Strickland, S., and
Tsirka, S.E. 1999. Activation of microglia reveals a non-proteolytic cytokine function for tissue plasminogen activator in the central nervous system. J. Cell Sci. 112:4007-4016.

15. Wu, Y.P., et al. 2000. The tissue plasminogen activator (tPA)/plasmin extracellular proteolytic system regulates seizure-induced hippocampal mossy fiber outgrowth through a proteoglycan substrate. J. Cell Biol. 148:1295-1304.

16. Nicole, O., et al. 2001. The proteolytic activity of tissue-plasminogen activator enhances NMDA receptor-mediated signaling. Nat. Med. 7:59-64.

17. Yepes, M., et al. 2002. Regulation of seizure spreading by neuroserpin and tissue-type plasminogen activator is plasminogen-independent. J. Clin. Invest. 109:1571-1578. doi:10.1172/JCI200214308.

18. Takao, M., et al. 2000. Neuroserpin mutation S52R causes neuroserpin accumulation in neurons and is associated with progressive myoclonus epilepsy. J. Neuropathol. Exp. Neurol. 59:1070-1086.

19. Strickland, S. 2001. Tissue plasminogen activator in nervous system function and dysfunction. Thromb. Haemost. 86:138-143.

20. Huang, Y.Y., et al. 1996. Mice lacking the gene encoding tissue-type plasminogen activator show a selective interference with late-phase long-term potentiation in both Schaffer collateral and mossy fiber pathways. Proc. Natl. Acad. Sci. USA. 93:8699-8704.

21. Frey, U., Muller, M., and Kuhl, D. 1996. A different form of long-lasting potentiation revealed in tissue plasminogen activator mutant mice. J. Neurosci. 16:2057-2063.

22. Madani, R., et al. 1999. Enhanced hippocampal long-term potentiation and learning by increased neuronal expression of tissue-type plasminogen activator in transgenic mice. $E M B O J$ 18:3007-3012

23. Pawlak, R., et al. Rapid, specific and active-site catalysed effect of tissue plasminogen activator in hippocampus-dependent learning. Neuroscience. In press

24. Salles, F.J., and Strickland, S. 2002. Localization and regulation of the tissue plasminogen activator-plasmin system in the hippocampus. J. Neurosci. 22:2125-2134. 